\title{
Family Physicians Ability to Perform Cesarean Sections Can Reduce Maternal and Infant Mortality
}

\author{
John Cullen, $M D$
}

( J Am Board Fam Med 2021;34:6-9.)

In the United States maternal and infant mortality are increasing. ${ }^{1}$ It is now more dangerous for our daughters to have children than for their mothers. Multifactorial reasons exist, including the greater maternal mortality for women of color as well as the increased mortality for pregnant women and neonates in rural communities. ${ }^{2}$ The decrease in the number of Family Physicians providing maternity care and performing cesarean sections may explain some of these trends.

The reality is that maternity care deserts are expanding, and this has led to higher maternal mortality for women in rural communities. The article by Tong et al on the essential role of Family Physicians providing cesarean sections in rural communities is timely and important. ${ }^{3}$ In true obstetric emergencies in rural communities, there is often not time to transfer patients, and geography or weather can complicate travel. However, moving to metropolitan areas weeks ahead of the due date can also be difficult and impractical. The latter is especially true for Black, Indigenous, and People of Color of lower economic status who are more challenged with difficulties of safe travel and staying in the larger community.

Having spent the past 26 years practicing fullscope rural Family Medicine including cesarean sections, I have found that even if a community is not planning on providing maternity care, they will continue to do so, albeit without the ability to handle

From the American Academy of Family Physicians, Leawood, KS

Funding: None.

Conflict of Interest: None.

Corresponding author: John Cullen, MD, American Academy of Family Physicians, 11400 Tomahawk Creek Pkwy, Leawood, KS 66211 (E-mail: johncullenmd@gmail.com).

See Related Article on Page 10. obstetric emergencies. Having successfully delivered 2 sets of preterm twins with prolapsed cords, I know from firsthand experience how quickly an obstetric emergency can develop in a rural setting even when the intention was to transfer care to a metropolitan area. Despite an intention of only attending low-risk births in our maternal population, I have had to perform emergency cesarean sections over the years, often with telemedicine consultation with Maternal Fetal Medicine Specialists. I would not have been able to save the lives of my patients without the cesarean skills I had learned in residency and then maintained in my community. Our communities' infant mortality $(3 / 10,000)$, cesarean section rate $(18 \%)$, and complications are lower than national averages. ${ }^{4}$ Aggressive risk stratification and the ability to do repeat and emergency Cesarean sections both contribute to these numbers.

Cesarean section skills not only translate to better outcomes in maternity care but also in trauma care and inpatient medicine. For a rural hospital, securing the ability to perform Cesarean sections translates to improved nursing skills and confidence, anesthesia capability, and increased training in programs such as neonatal resuscitation. Staff must often cross train and the increased emphasis of these skills on the health care team translates to improved care across hospital staff. The provision of high-quality maternity care increases community confidence in the quality of care at the hospital.

There is an economic argument as well. When Family Physicians with enhanced obstetrical skills were recruited to rural Alabama communities, they generated \$1.4 million dollars in indirect economic activity. ${ }^{5}$ Given the economic neglect of rural communities, especially those with large minority populations, which have the highest rates of maternal and infant mortality, investing in strong maternal 
care capability could address both issues. Improving training opportunities for rural Family Physicians to perform cesarean sections would be a cost-effective investment to improve rural economies.

Family physicians can perform Cesarean sections safely. ${ }^{6,7}$ Most importantly, they live and work within the communities where women of childbearing age live. We know that roughly a third of maternal mortality occurs during pregnancy, a third in the week surrounding delivery, and a third in the year after delivery. ${ }^{8}$ When women travel to deliver in metropolitan communities, the majority are discharged and back within their rural community within 48 hours. This means that the vast majority of maternal mortality occurs in the communities where pregnant women live, either during pregnancy or in the year after delivery. Every community must have a plan for obstetric emergencies and the ability of a rural hospital to handle obstetric emergencies by reaching the bar of offering safe Cesarean sections confers enormous advantages for the population it serves.

Women from rural communities who stay within their community to deliver have better outcomes than women who travel from rural communities to metropolitan areas to deliver. ${ }^{9}$ There are several potential reasons why these women have worse outcomes when traveling to metropolitan areas to deliver including increased stress in leaving family and social supports, a greater likelihood of induction, and a greater likelihood of Cesarean section. ${ }^{10}$ In addition, pregnant women are often either the primary source of childcare or of income (or both). Transferring care and staying in a metropolitan area until delivery disrupts familial and social supports, impacts employment, and reduces the amount of paid time spent at home after the delivery.

For these reasons, pregnant women stay at home within their community for as long as possible, and this trend increased with the onset of COVID-19 as pregnant women prefer to stay away from metropolitan areas. Some choose to have a home delivery, relying on the local hospital and physicians as a backup for complications even if they do not have the capability or expertise. Others wait until they are in labor to begin the trip to a larger community. As distances increase, the complication rate increases ${ }^{11}$ Women are less likely to receive prenatal care and more likely to deliver in inappropriate places such as Emergency Rooms or hospitals without obstetric capability. ${ }^{12}$
The loss of rural hospitals and maternity units increases the distance to maternity care. There have been 170 rural hospital closures since 2005 and many more in danger of closing: $12 \%$ of rural hospitals have closed their maternity units since 2011 and only $46 \%$ of rural hospitals provide obstetric services. ${ }^{13,14}$ Loss of rural hospital based obstetric services results in an increased risk of preterm birth. ${ }^{12}$

The solution to this to have maternal care capability within closer proximity to the rural communities where women of reproductive age live. The 2019 American College of Obstetricians and Gynecologists Levels of maternal care states,

"This information should not be interpreted to imply that hospitals with low delivery volumes are not safe for care of women with low-risk pregnancies, or as a call to close hospitals with a lower volume or acuity. In remote or rural areas, hospitals with low delivery volumes are often the only local delivery option. Rather, these data, combined with the fact that $59 \%$ of hospital births in the United States occur at hospitals where fewer than 1000 newborns are delivered annually, underscore the importance of adequately staffed and equipped level I and II hospitals; regionalized care with defined relationships between different level facilities; continuous risk assessment; and the potential benefit of caring for women with high risk of maternal morbidity in centers with higher level, acuity-focused resources and specialty and subspecialty personnel." ${ }^{15}$

The ability to perform an emergency Cesarean section is a prerequisite of modern obstetric care. As rural hospitals need to be able to provide maternity care, they must have Cesarean section capability. Although the Cesarean rate in the United States is excessive, the ability to perform one safely when needed is necessary.

Family Physicians with enhanced skills in obstetrics are the ideal workforce to address excessive rural maternal mortality. Because of their broad scope, Family Physicians do not need large volumes of obstetric patients to survive financially. In our frontier community of 4000 people, we can support 5 full-time Family Physicians with outpatient clinics, emergency department coverage, inpatient me-dicine, and procedures such as colonoscopy and upper endoscopy, in addition to maternity care.

OB/GYN prefer metropolitan and micropolitan practices and tend to not practice in rural noncore hospitals. ${ }^{16}$ The United States is also experiencing 
a shortage of $\mathrm{OB} / \mathrm{GYN}$ who deliver babies even within metropolitan and micropolitan areas. This suggests that other care providers are needed for obstetrics needs in rural communities. Certified Nurse Midwives are excellent partners for family physicians, but also are underrepresented in rural communities. ${ }^{16}$ Midwives require surgical backup, which Family Physicians can provide. General Surgeons can learn to do Cesarean sections, especially with onsite Family Physicians with obstetric experience as to when to do them, although they are also underrepresented in rural communities and do not have an obstetric background. ${ }^{17}$

Family Physicians are capable of performing Cesarean sections safely. There is a need for Family Phy-sicians to perform Cesarean sections, especially in rural communities. Having this capability would reduce maternal and infant mortality in those communities as well as providing a needed economic investment, especially in communities with a larger minority population. This capability would translate to a higher quality of care throughout the rural health care system and increased confidence in local hospitals. Even in communities where there are no plans for the provision of maternity care, women will continue to have obstetric complications in the communities where they live.

\section{Next Steps}

How do we increase the number of family physicians performing cesarean sections? First, we may need to increase the length of training for those who wish to offer this service. We must increase the number of rural and OB fellowships that offer enhanced surgical OB skills. It should be noted that there are residencies that provide adequate training in cesarean sections, Greely, Colorado and Via Christi in Kansas are just 2 such examples of 3-year programs whose graduates are providing needed care throughout rural America. ${ }^{18}$ For many Family Medicine graduates, a fellowship year is desired and may be necessary.

Second, academic institutions must recognize the importance of Family Medicine obstetrics and teach accordingly. There is a bias against primary care and Family Medicine within academic centers, a hidden curriculum, which has adversely affected both student choice and rural maternal and infant mortality. ${ }^{19}$ Teaching Hospitals should encourage qualified Family Physicians to have privileges to perform a cesarean section in their facilities to model what expanded scope family medicine looks like. At the very least they should not sabotage the dreams of medical students who want to go to a rural community, often returning to the communities they grew up in, to offer full-spectrum Family Medicine including Cesarean sections.

Third, regional health care systems should ensure that appropriate maternity care is offered in the rural communities in which they serve. Level III and IV levels of maternal care referral centers have an obligation of care for those communities that send them patients. Caring for these communities does not mean that all women need to deliver in the level III or IV facility. As mentioned earlier, this leads to worse outcomes and probably accounts for some of the observed increased maternal and infant mortality. Instead, higher levels of care should provide opportunities for consults, training in Alliance for innovation in Maternal Health (AIM) bundles, simulations, and providing mini fellowships so that rural family physicians can maintain their skills for low volume high acuity events. As stated in ACOGs Level of Maternal Care:

Regionalized maternal care is intended to maintain and increase access to care by developing, strengthening, and better defining relationships among facilities within a region. In turn, this should facilitate consultation and transfer of care when appropriate so that low- to moderate-risk women can stay in their communities while pregnant women with high-risk conditions receive care in facilities that are prepared to provide the required level of specialized care. ${ }^{15}$

Fourth, the barriers that impede qualified Family Physicians' ability to gain privileges for a Cesarean section must come down. This may take the education of our OB/GYN colleagues and may take additional certification such as a Certificates of Added Qualification in Family Medicine Obstetrics or Rural Family Medicine. Medical students and residents need to have Family Physician mentors who perform Cesarean sections.

Fifth, there needs to be further economic analysis of the impact of family physicians with Cesarean section skills on the financial health of rural hospitals. Too often administrators have a service line strategy where financial deficits in the maternity care unit prompts closure. If we are to improve maternal outcomes, additional payments and incentives must be given to keep maternal units open, especially within obstetric deserts. However, this 
economic analysis should not focus solely on obstetrics, but rather the economic and overall health outcomes on the hospital and the community as a whole. Family Physicians performing Cesarean sections not only means improved maternity care in rural communities, which leads to improved outcomes and decreases in maternal and infant mortality, but also more ability to care for nonobstetrical patients, more stable hospital financing, and healthier and more vibrant communities.

To see this article online, please go to: http://jabfm.org/content/ 34/1/6.full.

\section{References}

1. Lu MC. Reducing maternal mortality in the United States. JAMA 2018;320:1237-8.

2. Kozimannil KB, Interrante JD, Henning-Smith C, Admon LK.. Rural-urban differences in severe maternal morbidity and mortality in the US, 200715. Health Aff 2019;38:2077-85.

3. Tong ST, Eden AR, Morgan ZJ, Bazemore AW, Peterson LE. The essential role of family physicians in providing cesarean sections in rural communities. J Am Board Fam Med 2021;34:10-11.

4. Mead A. Strengthening the workforce to improve pregnancy outcomes in rural areas. Rural Health Information Hub July 11, 2018.

5. Avery D, Hooper DE, McDonald Jr JT, Love MW, Tucker MT, Parton JM. The economic impact of rural family physicians practicing obstetrics. J Am Board Fam Med 2014;27:602-10.

6. Deutchman M, Connor P, Gobbo R, FitzSimmons R. Outcomes of cesarean sections performed by family physicians and the training they received: a 15-year retrospective study. J Am Board Fam Pract 1995;8:81-90.

7. Homan FF, Olson AL, Johnson DJ. A comparison of cesarean delivery outcomes for rural family physicians and obstetricians. J Am Board Fam Med 2013;26:366-72.

8. CDC Vitalsigns. (2019, May). Retrieved from CDC. gov: www.CDC.gov/vitalsigns/maternal.deaths.

9. Nesbitt TS, Connell FA, Hart LG, Rosenblatt RA. Access to obstetric care in rural areas: effect on birth outcomes. Am J Public Health 1990;80:814-8.

10. Kornelsen J, Moola S, Grzybowski S. Does distance matter? Increased induction rates for rural women who have to travel for intrapartum care. J Obstet Gynaecol Can 2009;31:21-7.

11. Grzybowski S, Stoll K, Kornelsen J. Distance matters: a population-based study examining access to maternity services for rural women. BMC Health Serv Res 2011;11(147):1-8.

12. Kozhimannil KB, Hung P, Henning-Smith C, Casey MM, Prasad S. Association between loss of hospitalbased services and birth outcomes in rural counties in the United States. JAMA 2018;319:1239-47.

13. Tribble SJ. Prognosis for rural hospitals worsens with pandemic. Kaiser Health News August 26, 2020.

14. The decline of access to $\mathrm{OB}$ services in rural communities. Chartis Center for Rural Health February 2019.

15. ACOG. Levels of maternal care. Obstetric Care Consensus Number 9, August, 2019.

16. Patterson DG, Andrilla CHA, Garberson LA. The supply and rural-urban distribution of the obstetrical care workforce in the U.S. Policy brief \# 168. WWAMI rural health research center, University of Washington; June 2020.

17. Hung P, Kozhimannil KB, Casey MM, HenningSmith C, Prasad S. State variations in the rural obstetric workforce. Rural Health Research Center, University of Minnesota; May 2016.

18. Heider A, Neely B, Bell L. Cesarean delivery results in a family medicine residency using a specific training model. Fam Med 2006;38:103-9.

19. Mahood SC. Medical education: beware the hidden curriculum. Can Fam Physician 2011;57:983-5. 